\title{
Innovative gold nanoparticle patterning and selective metallization
}

\author{
E.S. Kooij ${ }^{1}$, M.A. Raza ${ }^{1,2}$, B. Poelsema ${ }^{1}$ and H.J.W. Zandvliet ${ }^{1}$ \\ ${ }^{1}$ Physics of Interfaces and Nanomaterials, MESA+ Institute for Nanotechnology, \\ University of Twente, P.O.Box 217, 7500AE, Enschede, The Netherlands, e.s.kooij@utwente.nl \\ ${ }^{2}$ Centre of Excellence in Solid State Physics, University of the Punjab, \\ QAC, Lahore-54590, Pakistan
}

\begin{abstract}
We present a simple procedure to selectively deposit gold nanoparticles using pure water. It enables patterning of nanoparticle monolayers with a remarkably high degree of selectivity on flat as well as microstructured oxide surfaces. We demonstrate that water molecules form a thin capping layer on exposed thiol molecules within mercaptan self-assembled layers. This reversible capping of water molecules locally deactivates the thiol groups, therewith inhibiting the binding of metallic gold nanoparticles to these specific areas. In addition, we show that this amazing role of water molecules can be used to selectively metallize the patterned gold nanoparticle arrays. Employing an electroless seeded growth process, the isolated seeds are enlarged past the percolation threshold to deposit conducting metal layers.
\end{abstract}

Keywords: patterning, gold nanoparticles, selective deposition, self-assembly, metallization

\section{INTRODUCTION}

In the fabrication of general purpose devices based on nanoparticles, one of the key challenges is the patterned deposition of the nanoparticles on pre-defined areas by simple, fast and low-cost methods. Various top-down and bottom-up techniques with sophisticated equipment and chemicals have been used to achieve nanoscale patterning. The majority of nano- and micropatterning techniques are in the former category, including photolithography, electron-beam lithography, microcontact printing, dip-pen nanolithography and laser-based patterning. Despite their enormous impact on modern technology, the top-down approaches have a number of disadvantages and limitations. For example photolithography and electron-beam lithography techniques require hazardous chemicals. Microcontact printing requires an elastomeric stamp to fabricate heterogeneous structures of typically micrometer dimensions, but use for a mixed functionality surface fabrication is limited [1]. In principle, dip-pen nanolithography enables nanoscale patterning [2]. However, the relativity low transfer efficiency hinders large scale fabrication.

The alternative bottom-up approaches resolve a number of drawbacks and limitations of the aforementioned methods. Moreover, various combinations of both 'topdown' and 'bottom-up' have also been suggested. An example of such a combined approach is referred to as microcontact deprinting [3] enabling hierarchical patterning of nanoparticles on a wide range of substrates. Although this is fast and versatile, it requires a plasma system to burn off the polymer micelles together with complicated procedures to apply and peel off the polymer stamp at high temperature therewith increasing the overall complexity.

Here we present a novel, simple, fast and low-cost method to enable patterned deposition of nanoparticles and demonstrate local metallization. In previous work we attempted to locally derivatize the surface with amino- and mercaptosilane molecules by microcontact printing [4]. Although there was an apparent density difference, the selectively was limited. The present approach does not require sophisticated equipment nor does it involve harsh chemical procedures.

\section{LOCAL NANOPARTICLE DEPOSiTION}

In Fig. 1 our method to pattern gold nanoparticle deposits is schematically shown; for specific experimental details, we refer to our previous work [5]. First, a cleaned $\mathrm{Si} / \mathrm{SiO}_{2}$ substrate is functionalized with mercaptosilane (MPTMS). In a next step, a specific area of the substrate is treated by bringing it into contact with pure water. Finally, gold nanoparticles are deposited by immersing the substrate into a nanocolloidal gold solution. In Fig. 2(center) a photograph of the sample after water treatment (bottom-right panel) using a droplet of millimeter dimension and subsequent gold nanoparticle deposition is shown. A circular spot can be identified where the water has been in contact with the MPTMS layer. A clear difference in reflective properties can be discerned between the areas of the surface which have been water-treated and the areas which have not been in contact with water. We assume that the difference in contrast in the macroscopic images arises from the fact that gold nanoparticles do not attach to the watertreated area, while the untreated surface is fully covered with nanoparticles.

To verify this assumption, typical electron microscopy 


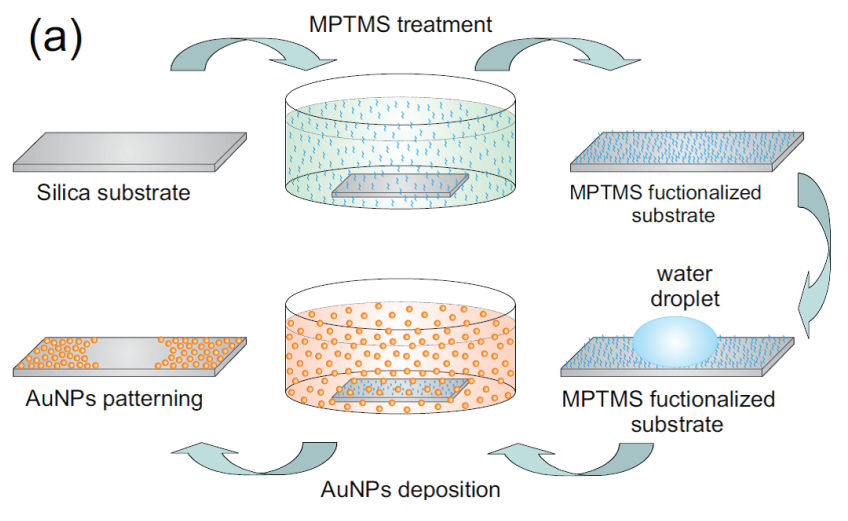

Figure 1: Schematic representation of the nanoparticle patterning using water. After derivatization of the surface with MPTMS, selective parts are defunctionalized by water treatment, followed by site-selective deposition of gold nanoparticles.

images are shown of silica coated with $50 \mathrm{~nm}$ gold nanoparticles at various places on the surface, as indicated in Fig. 2. The untreated area (top-right) shows a dense coverage of nanoparticles indicating the irreversible deposition of these nanoentities as described previously [6]. The strong interaction between the exposed thiol groups of the MPTMS molecules induces a strong affinity to irreversibly bind the gold nanoparticles to the surface. Surprisingly, on the area within the circle (top-left) where the MPTMS was in contact with the water droplet, gold nanoparticles do not adsorb. Remarkably, we did not even find a single gold particle, revealing the high degree of selectivity (bottom-left).

\section{PATTERNED FUNCTIONALIZATION}

To address the effect of water on the MPTMS-coated area, we have considered various possible scenarios. A first assumption involves removal of the layer of MPTMS from the water-treated area by washing off. This would explain why gold nanoparticles are not adsorbed in the water-treated areas since removal of MPTMS also leads to the absence of free thiol groups available for gold binding. A second possibility is that by water treatment the free thiol end-groups are oxidized to form sulfate ions. Therewith the functionality to irreversibly bind gold nanoparticles is lost. It is well-documented in literature that the presence of physisorbed water and oxygen at the MPTMS surface may lead to the oxidation of mercaptan [7-9]. In another possible oxidation process the thiol end groups are transformed into disulfide S-S entities with a low reactivity [10]. The replacement of thiol by disulfide groups could also account for the strongly reduced affinity to irreversibly bind gold nanoparticles.

In view of the aforementioned possible explanations for the effect of water on MPTMS monolayers, and to

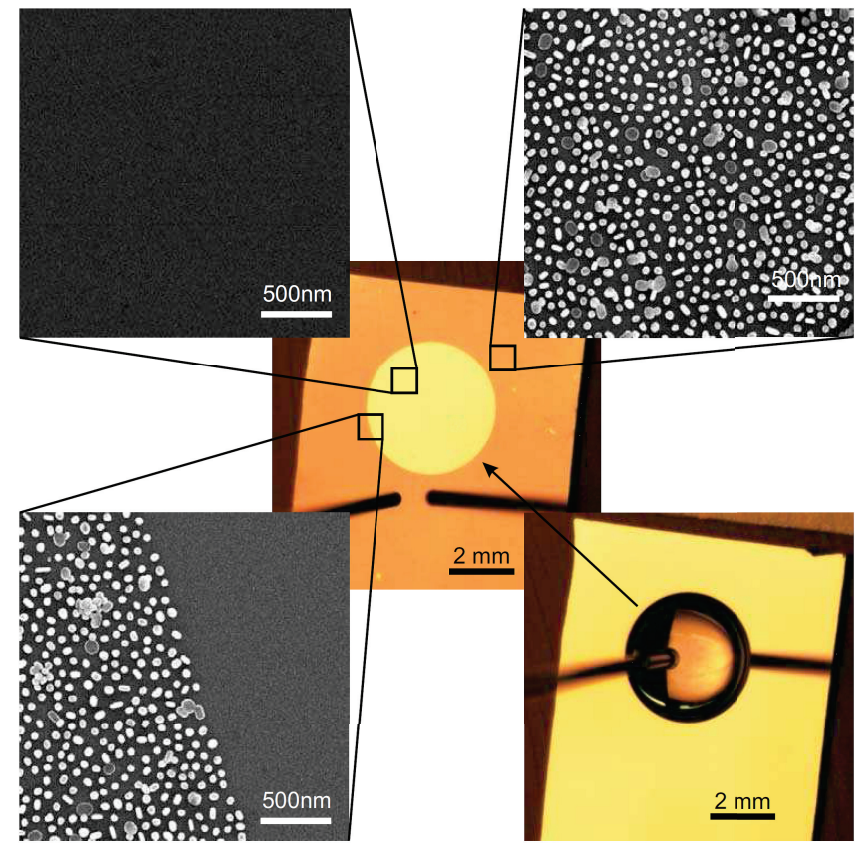

Figure 2: Macroscopic view and electron microscopic images of gold nanoparticle patterning on a flat $\mathrm{Si} / \mathrm{SiO}_{2}$ substrate. The bottom-right and center images are photographs during water treatment and after patterned nanoparticle deposition. Electron microscopy images show three areas: (i) non-water treated (top right), (ii) water-treated (top left), and (iii) border region [5].

elucidate the potential mechanism, we performed an essential experiment, which in fact rules out any of the aforementioned models. As described above, after water treatment the affinity to bind gold nanoparticles is completely destroyed. However, when samples are heated to approximately $120^{\circ} \mathrm{C}$ for a short period of time, typically 20 minutes, the functionality of the thiol molecules appears to be fully restored. As with non-water-treated samples, nanoparticle deposition is again possible after heating water-treated samples. In all cases, we did not observe any notable difference in deposition characteristics between situations (i) before water treatment and (ii) after water treatment and subsequent heating. This indicates the complete reversibility of the water treatment by simply heating the samples. None of the models described above can account for this observation. The washed-off MPTMS molecules are not restored during heating, while possibly oxidized states (either sulfate or disulfide entities) will not be restored to their reduced states simply by heating in an oxidizing environment.

An alternative explanation for the reversible disappearance of the gold binding functionality of the thiol groups follows from considering the electronegativities of oxygen, sulfur and hydrogen $(3.44,2.58$ and 2.2 , respectively) [11]. The differences between values for the constituting elements in water and thiol entities indicate 

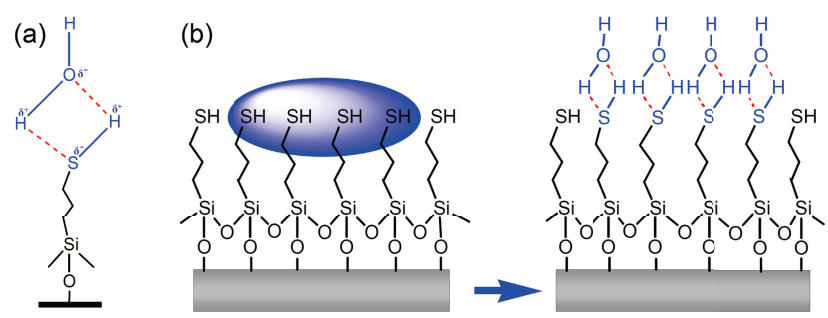

Figure 3: Schematic illustration (a) of the water molecule binding to the MPTMS molecule due to the electronegativity difference between $\mathrm{O}, \mathrm{S}$ and $\mathrm{H}$, and (b) the capping of water molecules shielding exposed thiol molecules, therewith hindering gold-sulfur bonding after water treatment on MPTMS and thus inhibiting gold nanoparticle deposition.

that both the $\mathrm{OH}$ and the $\mathrm{SH}$ bonds are polarized. As such, there will be an attractive electrostatic interaction between the water on one side and the thiol entities on the other. As such, we envisage that a bonding between the $\mathrm{OH}$ and $\mathrm{SH}$ bonds occurs (see Fig. 3), giving rise to hydrogen-bond type binding of a water monolayer to the MPTMS self-assembled monolayer.

Evidence for weak hydrogen bonds in thiols has also been derived from magnetic resonance experiments. Colebrook and Tarbell state that previous literature reviews indicate that thiol-based hydrogen bonds can be formed with strong donor groups such as oxygen. But they also suggest the possibility that the sulfur atom can act as a donor in hydrogen bonding [12]. In a more recent paper Yadav et al. [13] discuss the possible effect of water on the Michael addition. The proposed mechanism involves a double hydrogen bond between the water molecule and quinone compounds. The 'capping' of water molecules produces a thin layer of water on the thiol entities, which inhibits the effective binding of gold nanoparticles to the available sulfur groups on the surface. Simply stated, the water molecules effectively act as a 'resist' enabling patterning of whatever binds to the thiol-terminated MPTMS molecules.

This tentative mechanism would explain why gold nanoparticles are absent on water-treated regions. Also, it accounts for the restoration of the affinity to bind gold simply by heating the samples. Nevertheless, a surprising aspect of this tentative model is that in the case of non-water-treated samples, which exhibit a high affinity for gold, the nanoparticles are deposited from aqueous solutions. Why do gold particles not bind to previously water treated MPTMS layers, while they do adsorb in the presence of water?

\section{SELECTIVE METALLIZATION}

Following the localized gold nanoparticle deposition described in the previous section, selective metallization

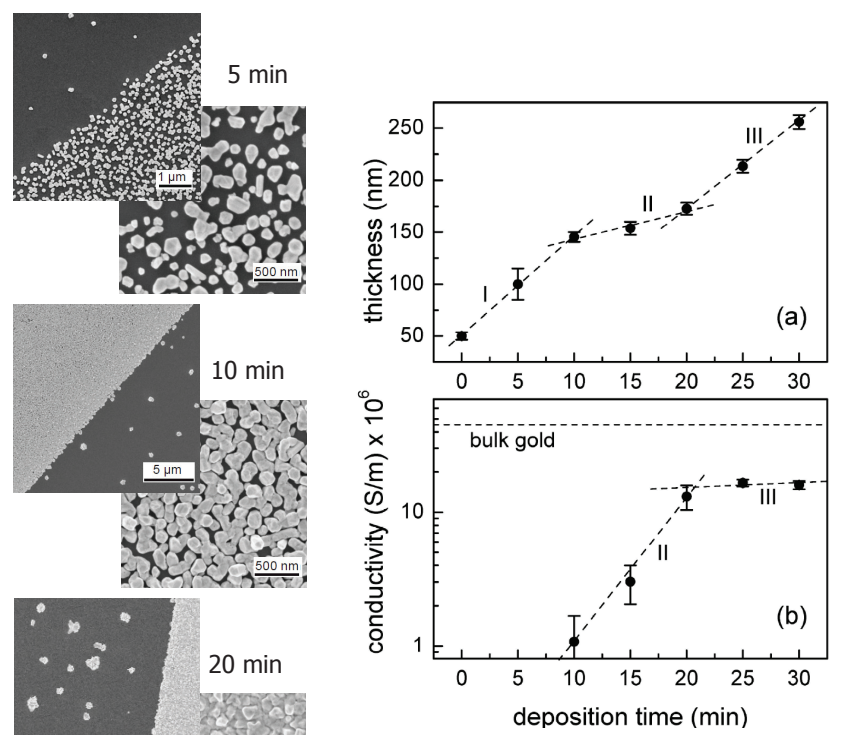

Figure 4: (left) Electron microscopy images showing the morphology of the samples for different electroless plating times after 5, 10 and 20 minutes. (right) Measured thickness (a) and conductivity (b) of electroless grown gold films as a function of plating time. Dashed lines are a guide to the eye; the dotted line in (b) represents the conductivity value for bulk gold [16].

can be achieved by electroless gold deposition on the patterned arrays which act as seeds for the electroless plating. A previously reported method was employed to grow continuous metal layers $[14,15]$. The growth solution essentially consists only of the precursor $\mathrm{HAuCl}_{4}$ and $\mathrm{NH}_{2} \mathrm{OH}$ as reducing agent. Typically within 20-30 minutes at room temperature, a continuous gold layer is formed specifically on the pre-patterned areas.

Electron microscopy images as shown in Fig. 4 of pre-patterned samples after seeded gold growth reveal a remarkable contrast between water-treated and nonwater-treated areas. The boundary between the different regions appears to be very sharp, clearly distinguishing between areas where gold nanoparticles were present and where they were absent. The electroless deposition process gives rise to enlargement of the isolated seed particles past the percolation threshold (a sequence of images is shown in the left part of Fig. 4), to ultimately give rise to thick, conducting gold films [16]. The morphology of the thick metallic layer in the nonwater-treated area is characterized by a large number of densely packed grains of different sizes. Owing to the absence of nanoparticles in the water-treated area such a metallic layer could not develop. However, after prolonged deposition, typically up to $30-40$ minutes, 
randomly distributed islands of different sizes in the low micrometer range develop within the water-treated areas; the initial stage is shown in the bottom-left image of Fig. 4. Our initial assumption was that these relatively large clusters form in solution and subsequently sediment onto the surface under the influence of gravity. However, changing the orientation of the substrate during seeded growth, even turning it upside down, reveals that the clusters are still formed. So apparently, these islands somehow nucleate within the defunctionalized regions, and grown into large entities with prolonged growth.

Further characterization and analysis of the electroless growth of gold on the patterned seed particle arrays reveals that the process is similar to what has been reported for silver growth $[4,17]$. Using a combination of optical tools (spectroscopic ellipsometry) and macroscopic conductivity experiments (right part of Fig. 4), we have identified different regimes during the seededgrowth. During the first few minutes, the electroless deposition gives rise to enlargement of the individual seed particles into large, but still isolated islands. Consequently, the macroscopic conductivity remains undetectably low. After approximately 10 minutes growth a percolating network of interconnected gold islands is formed, as is also confirmed by the fact that a finite, but small conductivity can be measured, which rises with growth time. Prolonged seeded growth eventually gives rise to a fully developed continuous layer (shown in the bottom-left image of Fig. 4). For films with a thickness up to a micrometer, the conductivity was found to saturate at approximately $30 \%$ of the bulk conductivity.

\section{CONCLUSIONS}

We show that pure water can be used to selectively defunctionalize MPTMS coated surfaces. We propose that water molecules form a thin 'capping' layer on exposed thiol molecules within the mercaptan layer. This reversible capping locally 'deactivates' the thiol groups, therewith inhibiting the binding of gold nanoparticles to these specific areas. The remarkably high degree of selectivity in the self-assembled formation of these nanogold monolayers is demonstrated. Since MPTMS is used as a binding agent not only for gold but also for other materials, the method described here can in principle be extended to enable patterning of other nanoscale materials.

In addition to the localized nanoparticle deposition, we studied the selective metallization by electroless gold deposition on the patterned seed particles. The latter gives rise to enlargement of the isolated seeds past the percolation threshold, to ultimately lead to conducting metal structures. The obtained patterned gold films exhibit macroscopic conductivity values approximately a factor of three lower than that of bulk gold.

\section{ACKNOWLEDGEMENTS}

The authors thank Dr. G. Hlawacek (University of Twente) for helpful discussions. One of the authors (M.A. Raza) acknowledges support from the Higher Education Commission in Pakistan.

Partly reprinted from J. Colloid Interface Sci. 364, M.A. Raza, E.S. Kooij, A. van Silfhout, H.J.W. Zandvliet, B. Poelsema, Novel, highly selective gold nanoparticle patterning on surfaces using pure water, pp. 304310. Copyright (2011), with permission from Elsevier.

\section{REFERENCES}

[1] L. Lioubelle, A. Bietsch, H. Schmid, B, Michel, E. Delamarche, Langmuir 15 (1999) 300

[2] R.D. Piner, J. Zhe, F. Xu, S. Hong, C.A. Mirkin, Science 283 (1991) 661

[3] J. Chen, P. Mela, M. Möller, M.C. Lensen, ACS Nano 3 (2009) 1451

[4] A.A. Mewe, E.S. Kooij, B. Poelsema, Langmuir 22 (2006) 5584

[5] M.A. Raza, E.S. Kooij, A. van Silfhout, H.J.W. Zandvliet, B. Poelsema, J. Colloid Interface Sci. 364 (2011) 304

[6] E.S. Kooij, E.A.M. Brouwer, H. Wormeester, B. Poelsema, Langmuir 18 (2002) 7677

[7] J. Singh, J.E. Whitten, J. Phys. Chem. C 112 (2008) 19088

[8] J.J. Senkevich, G.R. Yang, T.M. Lu, Colloids Surf. A 207 (2002) 139

[9] S.R. Yang, B.O. Kolbesen, Appl. Surf. Sci. 255 (2008) 1726

[10] G. Ledung, M. Bergkvist, A.P. Quist, U. Gelius, J. Carlsson, S. Oscarsson, Langmuir 17 (2001) 6056

[11] D.R. Lide (ed.), CRC Handbook of Chemistry and Physics, (Taylor \& Francis, Boca Raton, Fl., 2005)

[12] L.D. Colebrook, D.S. Tarbell, Proc. Natl. Acad. Sci. USA 47 (1961) 993

[13] J.S. Yadav, T. Swamy, B.V.S. Reddy, D.K. Rao, J. Mol. Catal. A: Chem. 274 (2007) 116

[14] K.R. Brown, L.A. Lyon, A.P. Fox, B.D. Reiss, M.J. Natan, Chem. Mater. 12 (2000) 314

[15] S.M. Tabakman, Z. Chen, H.S. Casalongue, H. Wang, H. Dai, Small 7 (2011) 499

[16] M.A. Raza, H.J.W. Zandvliet, B. Poelsema, E.S. Kooij, J. Appl. Phys. 113 (2013) 233510

[17] A.J. de Vries, E.S. Kooij, H. Wormeester, A.A. Mewe, B. Poelsema, J. Appl. Phys. 101 (2007) 053703 